\title{
ESTUDO DA EFICÁCIA TERAPEUTICA DO PRAZIQUANTEL (") E DAS ALTERAÇÕES MORFOLÓGICAS NOS OVOS DE HYMENOLEPIS NANA APÓS SUA ADMINISTRAÇÃO EM DOIS ESQUEMAS POSOLÓGICOS
}

\author{
Maria de Lourdes M. de CASTRo (1) e Getúlio Leonel de REZENDE (2)
}

\section{R E S UM O}

Foram selecionadas, numa comunidade fechada, 20 crianças com himenolepíase, apresentando carga parasitária acima de 1000 ovos $/ \mathrm{g}$ de fezes, visando a averiguar a eficácia terapêutica do praziquantel e as alteraçōes morfológicas induzidas nos ovos de H. nana por sua administração oral, em dois esquemas posológicos. Os pacientes foram alocados em dois grupos com igual número de casos. O grupo "A" foi tratado com uma única dose de $25 \mathrm{mg} / \mathrm{kg}$ de peso corporal e o grupo "B" com a mesma dose, porém repetida 10 dias depois. A partir do terceiro dia até o 19., verificou-se, em ambos os grupos, o surgimento, com um pico em torno do sétimo dia, de ovos distorcidos do parasita. Os ovos normais decresceram gradativamente, desaparecendo em $100 \%$ dos casos do grupo " $\mathrm{B}$ ", do 19: dia em diante, mas mantendo-se presentes em $20 \%$ dos casos no grupo " $\mathrm{A}$ ". A tolerância ao medicamento mostrou-se igualmente excelente com as duas posologias empregadas. Conclui-se pela elevada eficácia terapêutica do praziquan. tel na himenolepíase, sugerindo-se administrá-lo em duas doses de $25 \mathrm{mg} / \mathrm{kg}$, com um intervalo de dez dias, nos pacientes intensamente parasitados e que convivam em comunidades fechadas.

\section{N T RODU G Ã O}

As informações iniciais a respeito da eficécia do praziquantel (PZQ) sobre os cestóides datam de 1975, com as comunicações de THOMAS \& col.6,7. Em investigações posteriores, THOMAS 8 , utilizando ratos experimentalmen. te infectados com ovos de Hymenolepis nana, demonstrou que o $\mathrm{PZQ}$, em dose única de 25 $\mathrm{mg} / \mathrm{kg}$, provocava a eliminação de todos os parasitas localizados na luz intestinal. Ficou, também, comprovado que quanto maior o grau de maturação do parasita, tanto mais ativo era o PZQ. Com efeito, sua atividade sobre os cisticercóides com 24-48 horas de evolução, enquistados nas vilosidades intestinais, era muito menor, aumentando significativamente sobre as larvas com mais de 96 horas. Esse fato, segundo o Autor mencionado, decorria da falta de diferenciação orgânica do parasita nessa fase evolutiva inicial.

Observações realizadas in vitro ${ }^{1,9}$ permitiram constatar que, logo após o contato com o PZQ, o verme sofre uma forte contração tônico-clônica e torna-se imobilizado. Subseqüentemente, ocorrem modificações estruturais no tegumento, mais nítidas na região do pescoço (área de intenso metabolismo), conseqüentes à ação de enzimas proteolíticas. As lesões, quase sempre irreversíveis, constam inicialmente de vesículas que em seguida confluem, formando vacúolos. Posteriormente, verifica-se um

(*) Cestox - Merck S. A. Indústrias Químicas

(1) Mestre em Gastrenterologia pela Pontifícia Universidade Católica do Rio de Janeiro, RJ, Brasil

(2) Médico responsável pelo Dpto. de Pesquisa Clínico-Farmacológica da Merck S. A., Rio de Janeiro, RJ Trabalho apresentado no XVIII Congresso Panamericano de Gastroenterologia, Guatemala, novembro de 1983 
CASTRO, M. de L. M. de \& REZENDE, G. L. de - Estudo da eficácia terapêutica do praziquantel e das alterações morfológicas nos ovos de Hymenolepis nana após sua administração em dois esquemas posológicos. Rev. Inst. Med. trop. São Paulo 27:40-45, 1985.

derrame sincicial, acarretando a completa deseneração do tegumento.

Recentemente, procurando determinar o grau de atividade do $P Z Q$ nas diferentes fases do desenvolvimento do H. nana, CAMPOS $\&$ col. ${ }^{2}$, após infectarem com ovos desse parasita diversos lotes de camundongos, notaram que, a partir do $100^{\circ}$ dia de sua evolução, quando já tinham atingido a maturidade mas ainda não haviam iniciado a postura de ovos, ocorria a destruição de $100 \%$ dos vermes. Esses Autores depreenderam que tal fato poderia explicar as eventuais falhas terapêuticas quando se administrava uma única dose do PZQ. Assim, os cisticercóides alojados no interior das vilosidades e não atingidos pela ação medicamentosa, ao cabo de 10-14 dias, desenvolverse-íam em vermes adultos, reiniciando o ciclo parasitário. Pela mesma dedução justificam a đificuldade na erradicação na himenolepíase em comunidades fechadas e sugerem, nessa eventualidade, o uso do PZQ em duas doses administradas com 10 dias de intervalo.

CASTRO \& col. 4 , utilizando o esquema posológico proposto por CAMPOS \& col. ${ }^{3}$, trataram 51 individuos com himenolepíase, vivendo em um orfanato, e alcançaram $100 \%$ de índice de cura. Constataram, ademais, nos controles parasitológicos efetuados nos $7 .^{\circ}$ e $14 .^{\circ}$ dias pós-tratamento, o encontro de ovos do H. nana que se apresentavam inteiramente distorcidos. Levantaram, então, a hipótese de ter o PZQ atuado sobre os órgãos reprodutores daqueles parasitas que estavam em fase terminal de maturaçâo, prestes a iniciarem a oviparidade.

Com o intuito de investigar essa hipótese, elaboramos este estudo, objetivando observar a eficácia terapêutica do $\mathrm{PZQ}$ e o comportamento dos ovos de H. nana após a administração do medicamento em dois esquemas posológicos.

\section{PACIENTES E METODOS}

Numa comunidade fechada selecionamos 20 crianças com himenolepíase que apresentavam, pelo menos, 1000 ovos/g de fezes, segundo exame pela técnica quantitativa de KATO/ KATZ 5. A escolha de pacientes numa coletividade e com esse limite mínimo de carga parasitária baseou-se na pressuposição de, pela. possibilidade de reinfecções frequientes e pela presença de um parasitismo intenso, serem portadores de H. nana em diversos estágios evolutivos.

A casuística, Quadro I, constituía por 15 meninos e cinco meninas, em idade pré-escolar (na média 4 anos e 7 meses), foi distribuída em dois grupos, " $A$ " e " $B$ ", com igual número de casos, a fim de permitir a comparação de dois esquemas posológicos.

Q U A D R O I

Características dos pacientes com himenolepíase tratados com praziquantel

\begin{tabular}{|c|c|c|}
\hline Grupo & A & B \\
\hline Idade (anos) & N. ${ }^{\circ}$ de casos & N.o de casos \\
\hline 3 & - & 2 \\
\hline 4 & 3 & 6 \\
\hline 5 & 3 & 2 \\
\hline 6 & 4 & - \\
\hline Média & 5 anos ( \pm 3 meses) & 4 anos $( \pm 2$ meses $)$ \\
\hline Sexo & N.' de casos & N.o de casos \\
\hline Masculino & 5 & 10 \\
\hline Feminino & 5 & - \\
\hline Carga parasitária & N.o de ovos/g & N. ${ }^{\circ}$ de ovos/g \\
\hline Minima & 1392 & 1176 \\
\hline Máxima & 11712 & 13368 \\
\hline Média & $5081 \quad( \pm 1256)$ & 4039 ( \pm 1271$)$ \\
\hline
\end{tabular}

( ) = Desvio padrão da média

O maior número de crianças do sexo mas. culino habitando essa comunidade foi o res ponsável por sua prevalência na população estudada. Ambos os grupos eram homogêneos ( $\mathrm{p}<0,1$ - teste U de Mann-Whitnex) quanto à carga parasitária inicial.

O PZQ foi empregado por via oral, na dose de $25 \mathrm{mg} / \mathrm{kg}$ de peso corporal, administrada uma só vez ao grupo "A" e duas vezes, com um intervalo de 10 dias, ao grupo " $B$ ". Com a finalidade de afastar o risco de reinfecção durante o período de controle parasitológico, o que prejudicaria a avaliação dos resultados, to dos os indivíduos da comunidade também fo. ram tratados com uma dose de $25 \mathrm{mg} / \mathrm{kg}$. 
O acompanhamento parasitológico foi efetuado mediante exames coproscópicos seriados. As amostras de fezes colhidas diariamente, desde o dia seguinte à tomada do medicamento, eram conservadas em solução de mertiolato, iodo e formol (MIF). A cada dois dias, a partir do primeiro dia após o tratamento, realizava-se o exame (em três amostras) por microscopia direta, procurando-se não apenas detectar a presença de ovos do H. nana, como também observar o aspecto morfológico dos mesmos. O Grupo "A" foi controlado até o 21 . dia e o " $B$ " até o 31 .

$\mathrm{Na}$ avaliação da eficácia terapêutica foram considerados os resultados das coproscopias, quer em relação à positividade, quer à normalidade dos ovos.

\section{RESULTADOS}

Conforme visualizado nos Gráficos 1 e 2, a partir do terceiro dia seguinte à tomada do $P Z Q$, começam a surgir, em ambos os grupos, ovos distorcidos de H. nana que não haviam sido observados antes do tratamento. Em torno do sétimo dia eles estavam presentes em $80 \%$ dos casos e do nono dia em diante, esse porcentual reduziu-se, para cair a zero no 21. dia. Os gráficos mostram, ainda, que a porcentagem de pacientes com ovos normais nos exames de fezes posteriores ao tratamento sofre uma queda progressiva. Contudo, no grupo "A" persiste uma positividade residual em $20 \%$ dos casos. Já no " $\mathrm{B}$ " todos os pacientes ficam negativados do 19.' dia em diante. Resulta, portanto, um índice de cura de $80 \%$ e de $100 \%$, respectivamente nos grupos "A" e "B".

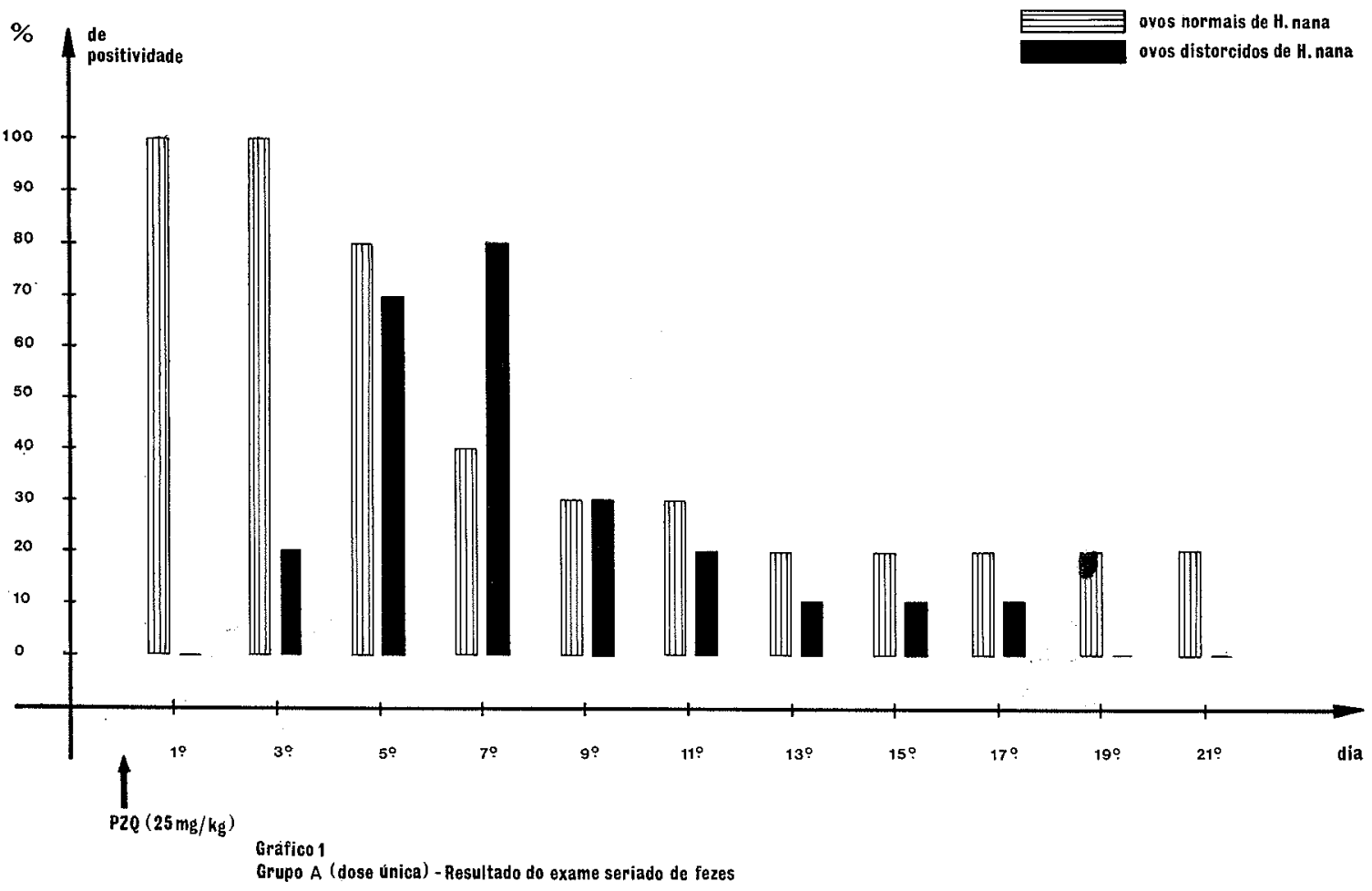

Pelo Quadro II, em que se correlaciona o resultado do tratamento com o número inicial de ovos normais detectados em cada caso, nota-se que os dois únicos pacientes não curados, ambos pertencentes ao grupo " $\mathrm{A}$ ", eram justamente os com maior carga parasitária.
A tolerabilidade do PZQ mostrou-se igual. mente satisfatória, com um ou com o outro esquema posológico, não se tendo registrado a ocorrência de qualquer tipo de efeito colateral. 
CASTRO, M. de L. M. de \& REZENDY̌, G. L. de - Estudo da eficácia terapêutica do praziquantel e das alterações morfologicas nos ovos de Hymenolepis nana após sua administração em dois esquemas posológicos. Rev. Inst. Med. trop. São Paulo $27: 40-45,1985$.

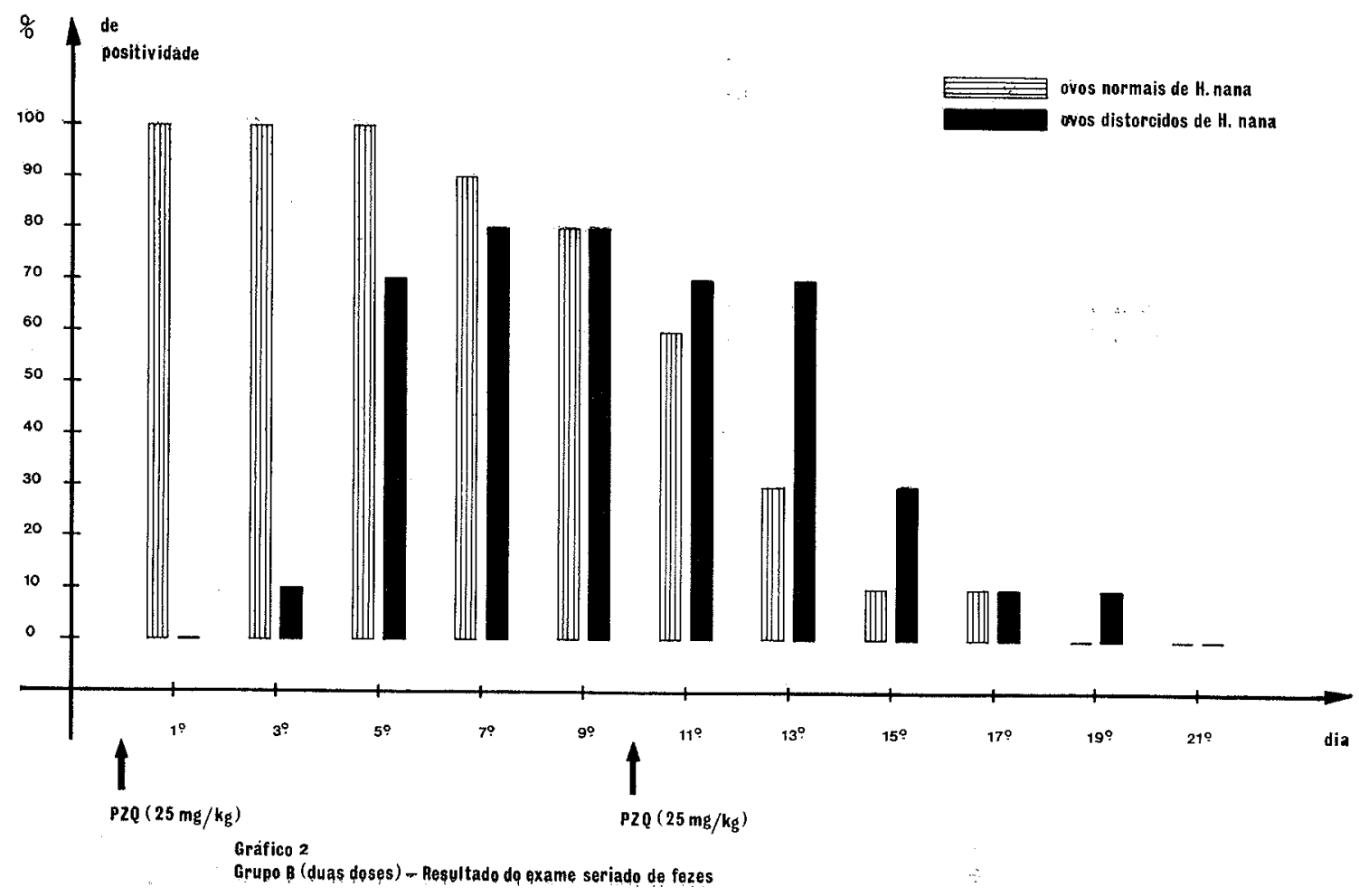

Q U A D R O I I

Resultado do tratamento em função da intensidade do parasitismo inicial em cada caso

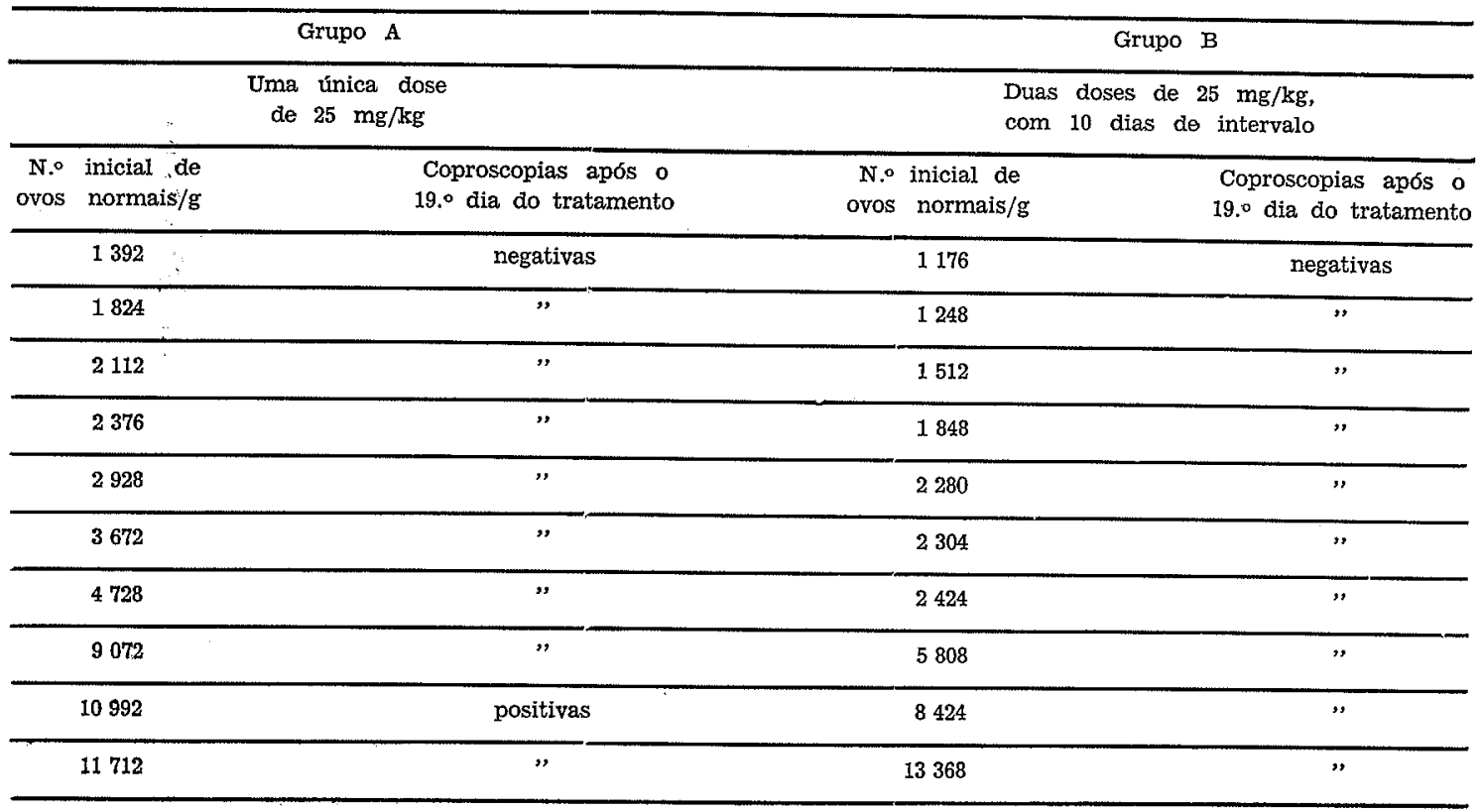

Parasitismo concomitante ficou evidencia- tro, tricocefalose em três e ambas as infestado em 11 dos 20 pacientes: ascaridíase em qua- ções em quatro. A presença desses vermes não 
CASTRO, M. de L. M. de \& REZENDE, G. L. de - Estudo da eficácia terapêutica do praziquantel e das alteraçöes morfológicas nos ovos de Hymenolepis nana após sua administração em dois esquemas posológicos. Rev. Inst. Med. trop. São Paulo 27:40-45, 1985.

exerceu influência alguma sobre o resultado da medicação. Além dessas, nenhuma outra patologia foi diagnosticada associada à himenolepíase.

\section{COMENTARIOS}

O aparecimento, exclusivamente e por um curto período, em seguida ao tratamento, de ovos distorcidos de H. nana, configurando uma distribuição semelhante a uma curva normal assimétrica, corrobora nossa hipótese de que - PZQ atue sobre o parasita em fase final de maturação, induzindo-o a formar ovos degenerados. Inclusive, o número de pacientes em que esses ovos foram encontrados até o nono dia é similar nos dois grupos (20 no "A" e 24 no "B"). Entretanto, torna-se dispar (5 e 14 Jespectivamente) do $110^{\circ}$ dia em diante. Esse fato parece indicar que a segunda dose administrada ao grupo " $B$ " desencadeou, novamente, aquele processo, aumentando a frequiência e duração de sua ocorrência.

A constatação de que as duas falhas terapêuticas ocorreram em pacientes tratados com uma única dose e portadores das cargas parasitárias mais elevadas está consoante com a suposição de que os casos mais intensamente parasitados sejam aqueles que mais provavelmente alberguem o H. nana, em diferentes estágios evolutivos, inclusive nas formas cisticercóides, relativamente imunes ao PZQ. Assim, explicar-se-ía o motivo dessas falhas terapêticas. Por outro lado, no grupo " $B$ ", com o emprego de duas doses, todos os pacientes foram curados, independentemente da intensidade do parasitismo inicial. Deduz-se, portanto, que as formas larvares que possam ter escapado à primeira dose, desenvolvendo-se em vermes maduros por ocasião da segunda administração, 10 dias depois, não mais foram capazes de resistir à ação medicamentosa.

Consequientemente, nos pacientes com carga parasitária elevada e que habitem em comunidades fechadas está indicado o tratamento com duas doses, com um intervalo de 10 dias. No entanto, nos demais casos, que constituem a grande maioria, é suficiente o emprego de uma única dose.

Ressaltamos, ainda, que para o critério de cura parasitológica da himenolepíase não se deveria considerar a simples presença de ovos nos exames de fezes realizados anteriormente ao $21^{\circ}$ dia subseqüente ao tratamento. E necessário, também, proceder-se a diferenciação entre ovos normais e distorcidos, pois, sendo encontrados exclusivamente os últimos, esse achado não correponde a uma falha terapêutica.

\section{SUMMARY}

Investigation of the therapeutical efficacy and the morphological alterations induced on the Hymenolepis nana eggs, administering praziquantel in two dose schedules

Twenty children with hymenolepiasis, living at a close community and having more than $1000 \mathrm{eggs} / \mathrm{g}$ of feces, were selected for the investigation of the therapeutical efficacy and the morphological alterations induced on the H. nana eggs, following the oral administration of praziquantel in two posologies.

The patients 'were allocated into two groups having equal number of cases. Group " $A$ " was treated with a single dose of $25 \mathrm{mg} / \mathrm{kg}$ BWT and group " $\mathrm{B}$ " with the same dose but repeated 10 days afterwards.

From the third day on until the 19th day it was found, in both groups, the uprising, with a peals around the seventh day, of distorted eggs. The normal eggs decreased gradually. They disappeared in $100 \%$ of the cases in group " $B$ ", from the 19th day on, but remained present in $20 \%$ of the cases in group "A".

The tolerance to the drug was excellent with either dose schedule.

It was concluded that praziquantel is highly efficaceous in the treatment of hymenolepiasis and that this drug should be administered in two doses of $25 \mathrm{mg} / \mathrm{kg}, 10$ days apart, for those patients presenting intense worm burden and living in close communities.

\section{REFERENCIAS BIBLIOGRAFICAS}

I. BECKER. B.; MEHLHORN, H.; ANDREWS, P. \& THOMAS, H. - Scanning and transmission electron microscope studies on the efficacy of praziquantel on Hymenolepis nana (Cestoda) in vitro. Z. Parasitenkd. 61: 121-133, 1980.

2. CAMPOS, R.; VIEIRA BRESSAN, M. C. R. \& EVANGELISTA, M. G. B. E- Estudos sobre a ação do 
CASTRO, M. de L. M. de \& REZENDE, G. L. de - Estudo da efiććcia terapêutica do praziquantel e das alteraçóes morfológicas nos ovos de Hymenolepis nana após sua administração em dois esquemas posológicos. Rev. Inst. Med. trop. São Paulo 27:40-45, 1985.

praziquantel em camundongos experimentalmente infestados com Hymenolepis nana. VI Congresso da Federação Latino-Americana de Parasitólogos, São Paulo, Bra. sil, 1983.

3. CAMPOS, R.; MOREIRA, A. A. B.; PINTO, P. L.; AMATO NETO, V.; LEITE, R. M. \& SANT'ANA, E. J. - Tentativa de controle de himenolepíase em coletividade semifechada. VI Congresso da Federação LatinoAmericana de Parasitólogos, São Paulo, Brasil, 1983.

4. CASTRO, M. L. M.; MACHado E SILVA, J. R. \& SAMPAIO, J. I. M. - Tentativa de erradicação com praziquantel da himenolepíase em uma comunidade semifechada. Rev. Inst. Med. trop. São Paulo 27: 27-32, 1985.

5. KATZ, N.; CHAVES, A. \& PELLEGRINO, J. - A simple device for quantitative stool thick-smear thecnique in Schistosomiasis mansoni. Rev. Inst. Med. trop. São Paulo 14: 397-400, 1972.
6. THOMAS, H.; GONNERT, R.; POHLKE, R. \& SEU. BERT, J. - Investiga'ions about treatment of larval cestodes. VII International Conference on Pathophysio. logy of Parasitic Infections. Tessalonica, Grécia, 1975.

7. THOMAS, H.; GÖNNERT, R.; POHLKE, R. \& SEU. BERT, J. - A new compound against adult tapeworms. VII International Conference on Pathophysiology of Parasitic Infections. Tessalonica, Grécia, 1975.

8. THOMAS, H. - Resultados experimentales con praziquantel en cestoidiasis y cisticercosis. Bol. Chile. Pa. rasit. 32: 2-6, 1977.

9. THOMAS, H. \& GÖNNERT, R. - The efficacy of praziquantel against cestodes in animals. Z. Parasitenkd. 52: $117-127,1977$.

Recebido para publicação em $3 / 4 / 1984$.

Endereço do Autor: Rua Teodoro da Silva, 712 - apto. 802 20.560 - Rio de Janeiro, RJ - Brasil 